\title{
October 2014 Imaging Case of the Month
}

Sameh Sakla, M.D.

Clinton Jokerst, M.D.

Department of Medical Imaging

University of Arizona Medical Center

Tucson, AZ

A 53-year-old man presents with fatigue and dyspnea on exertion. An admission chest radiograph (Figure 1) was obtained.

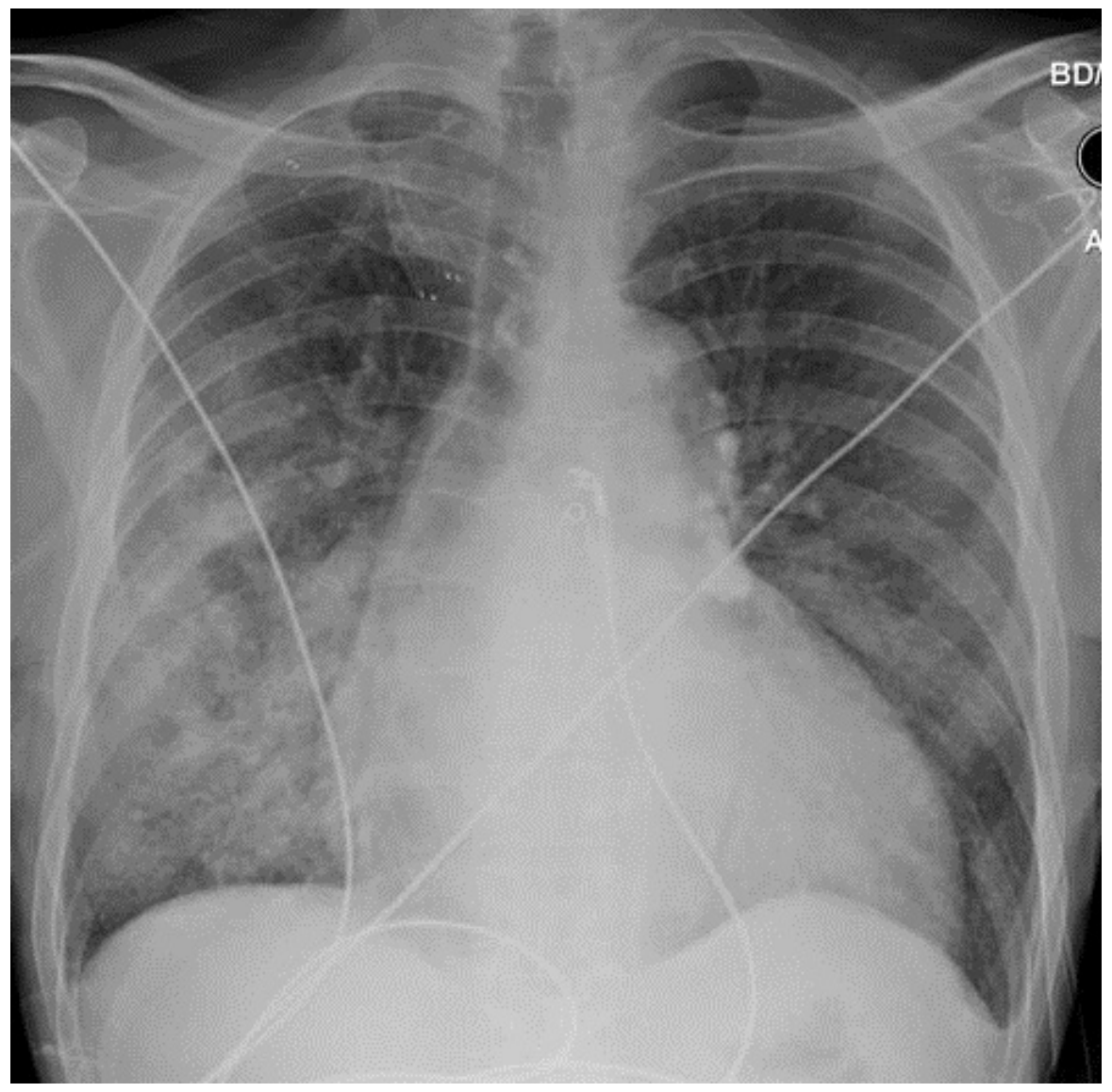

Figure 1. Admission chest radiograph.

What is the best term or phrase used to describe the salient radiographic abnormality?

1. Diffuse thick-walled cavitary lesions

2. Interstitial and alveolar pulmonary edema with effusions

3. Miliary nodules

4. Patchy consolidation

5. Tension pneumothorax 


\section{Correct! \\ 4. Patchy consolidation}

Posteroanterior chest radiograph demonstrates patchy airspace disease predominately in the form of right middle and right lower lobe consolidation. Findings are most concerning for pneumonia. Patient also has cardiomegaly suggesting heart disease or volume overload. A right subclavian vein stent suggests underlying renal disease.

A thoracic CT was performed for further evaluation (Figure 2).

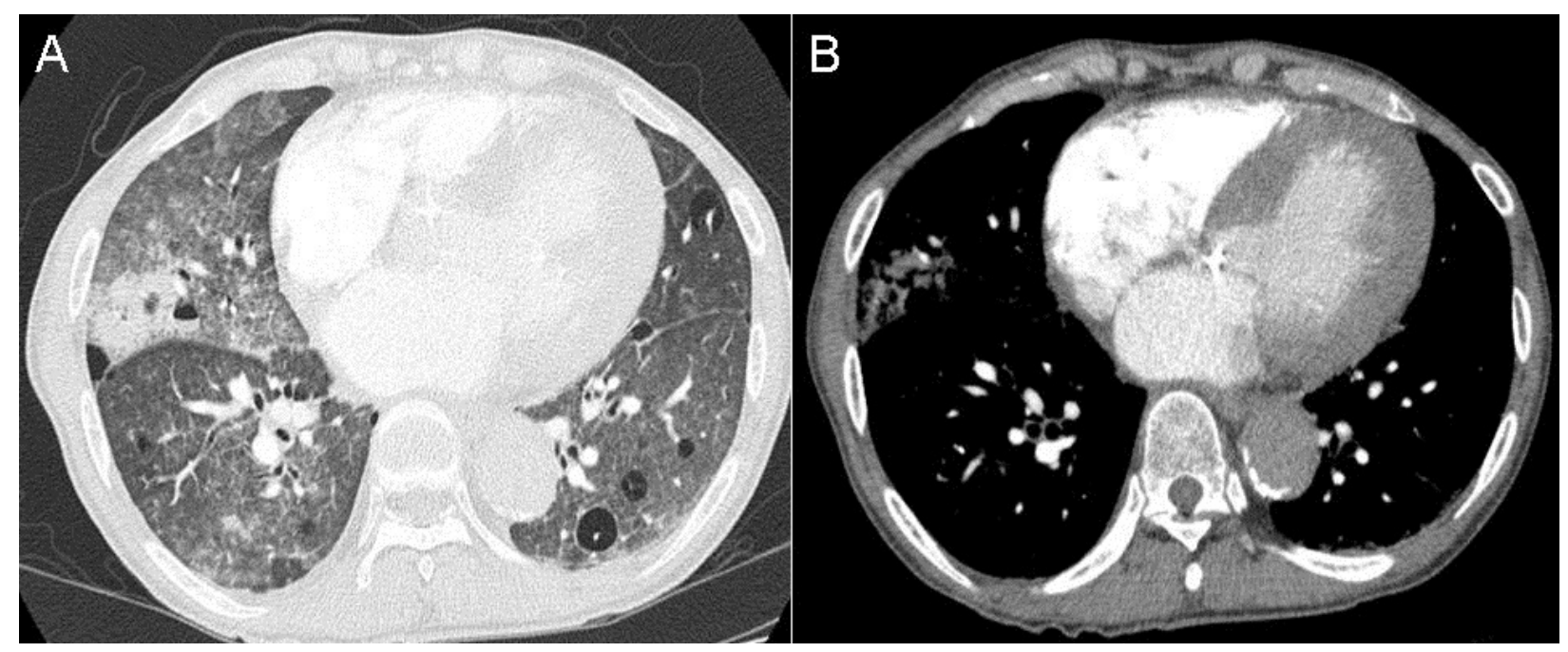

Figure 2. Panel A: representative lung window. Panel B: representative soft tissue window.

In addition to right middle lobe and right lower lobe consolidation/pneumonia, multiple pulmonary cysts were noted. Many of these cysts are associated with calcified pulmonary nodules. In addition, the left ventricle is diffusely thickened.

Given the finding of pulmonary cysts, which of the following diagnoses is least likely favored as part of the differential diagnosis in this patient?

1. Langerhan's cell histiocytosis (LCH)

2. Lymphangioleiomyomatosis (LAM)

3. Lymphocytic Interstitial pneumonia (LIP)

4. Pulmonary nodular amyloidosis

5. Pulmonary sarcoidosis 


\section{Correct!}

\section{Pulmonary sarcoidosis}

Pulmonary cysts are associated with answers 1-4 but are not typically seen in sarcoidosis (5). Pulmonary sarcoidosis typically presents with mediastinal adenopathy and perilymphatic micronodules, although it can present with large scattered nodules (nummular sarcoid). Sarcoid rarely cavitates.

LIP is a rare interstitial lung disease that is often seen in association with Sjogren's syndrome. Common pulmonary manifestations include lower-lobe predominant ground glass opacities and pulmonary cysts. Pulmonary nodular amyloidosis is a form of systemic amyloidosis where nodular collections of amyloid collect in the lungs. These nodules are of varying sizes and can calcify. Often there are cysts associated with these nodules (as in this case).

Amyloidosis is often a systemic process that can affect any organ system. The myocardial thickening seen on this patient's CT could be a cardiac manifestation of amyloidosis. LAM typically affects women of childbearing age and has overlap with tuberous sclerosis. Classically, the patients develop innumerable pulmonary cysts of relatively uniform size and distribution. In pulmonary LCH the cysts are often interspersed with nodules and have an upper lung predilection with sparing of the costophrenic angles. LCH is often associated with cigarette smoking and usually improves with smoking cessation.

Biopsy of the patient's pericardial fat pad demonstrated areas of classic apple-green birefringence with Congo red staining consistent with amyloid deposition.

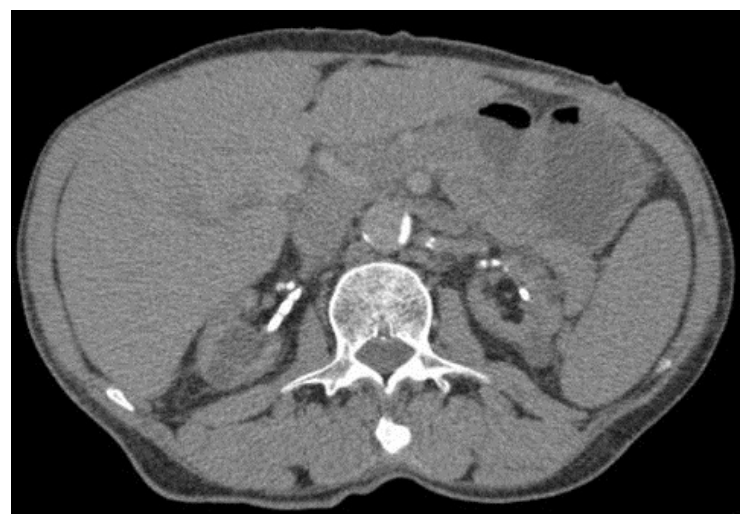

Figure 3. Representative upper abdominal CT image.

Based on the above image (Figure 3), classify the type of amyloidosis in this patient.

1. Familial amyloidosis

2. Primary amyloidosis

3. Secondary amyloidosis

4. None of the above 


\section{Correct!}

\section{Secondary amyloidosis}

Figure 3 shows bilateral atrophic kidneys consistent with chronic renal disease indicating secondary amyloidosis as the best answer. Secondary amyloidosis is characterized by deposition of an acute phase protein due to chronic infection and/or inflammation and usually affects the kidneys first. The patient did not have a history of multiple myeloma and therefore, primary amyloidosis, which is characterized by amyloid light chain deposition, is not the correct answer. Additionally, familial amyloidosis is identified as a genetic mutation responsible for the coding and production of the protein transthyretin; no information obtained by the history or the image points to familial amyloidosis as the correct answer.

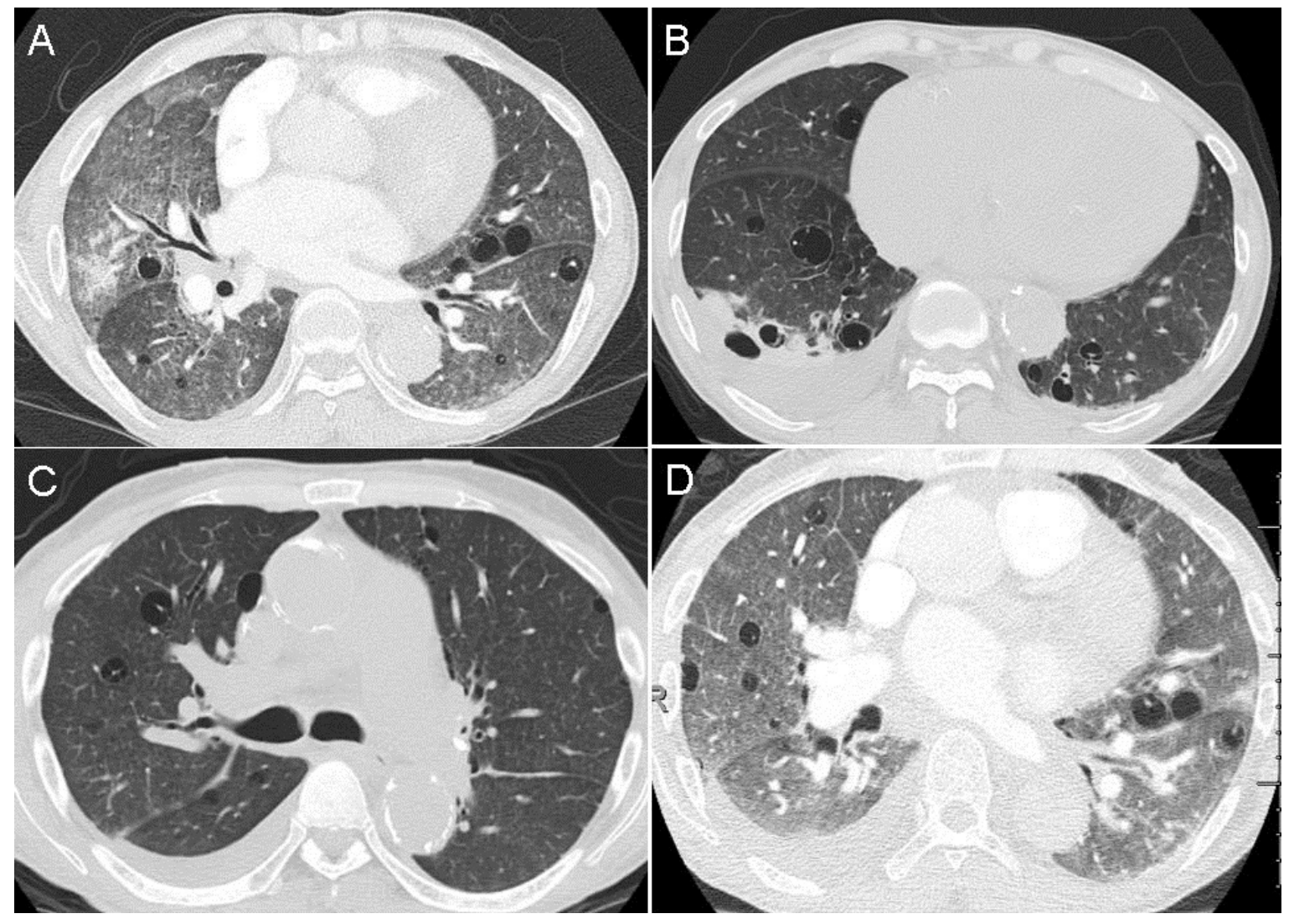

Figure 4. Representative images from serial CT scans. Panel A: The initial chest CT Panel B: 8 weeks after the initial chest CT. Panel C: 12 weeks. Panel D: 5 months.

Which of the following choices most closely characterizes the association between the type of pulmonary amyloidosis and the entity associated with it?

1. Diffuse parenchymal, Langerhan's cell histiocytosis

2. Diffuse parenchymal, lymphocytic interstitial pneumonia

3. Nodular, lymphocytic interstitial pneumonia

4. Tracheobronchial, lymphangioleiomyomatosis 


\section{Correct! \\ 3. Nodular, lymphocytic interstitial pneumonia}

Larger pulmonary parenchymal nodules are seen with the nodular type of pulmonary amyloidosis $(0.5-5.0 \mathrm{~cm}$ nodules in size). The tracheobronchial type of pulmonary amyloidosis has no known association with LAM and therefore, choice 1 is incorrect. Diffuse parenchymal nodules can occur in the lungs and are usually seen in the setting of smaller micronodules (2-4 mm). However, previously described case reports in the literature have identified a relationship between lymphocytic interstitial pneumonia (LIP) and amyloidosis. Furthermore, in some cases, cystic lesions may be not solely be on the basis of amyloidosis but also due to concomitant LIP. Therefore, the best answer is 3.

\section{References}

1. Lee AY, Godwin JD, Pipavath SNJ. Case 182: Pulmonary amyloidosis. Radiology 2012; 263(3):929-32. [CrossRef] [PubMed]

2. Urban BA, Fishman EK, Goldman SM, Scott WW Jr, Jones B, Humphrey RL, Hruban RH. CT evaluation of amyloidosis: spectrum of disease. RadioGraphics 1993;13(6):1295-1308. [CrossRef] [PubMed]

3. Georgiades CS, Neyman EG, Barish MA, Fishman EK. Amyloidosis: review and CT manifestations. Radiographics 2004;24(2):405-16. [CrossRef] [PubMed]

4. Boston University Amyloidosis Center. Amyloid types. Available at: http://www.bu.edu/amyloid/about/what/types/ (accessed 10/2/14). 